\title{
EDITORIAL
}

\section{Winners of the 2015 JA Medals for excellence}

\author{
The Journal of Antibiotics (2016) 69, 657-659; doi:10.1038/ja.2016.96
}

$\mathrm{T}$ he Editorial Board of The Journal of Antibiotics has awarded the 2015 JA Medal for an original article to an outstanding paper entitled 'MS/MS-based networking and peptidegenomics guided genome mining revealed the stenothricin gene cluster in Streptomyces roseosporus' by Wei-Ting Liu, Anne Lamsa, Weng Ruh Wong, Paul D Boudreau, Roland Kersten, Yao Peng, Wilna J Moree, Brendan M Duggan, Bradley S Moore, William H Gerwick, Roger G Linington, Kit Pogliano and Pieter C Dorrestein from University of California, San Diego and Santa Cruz. ${ }^{1}$ In this article, the authors report a global visualization of the detectable molecules produced from a single microorganism by MS/MS molecular networking, which allowed identification of the stenothricin gene cluster in combination with peptidogenomics.

MS/MS networking is a method of mapping a network of secondary metabolites, wherein molecules that are characterized by MS are subjected to fragmentation, and the resulting patterns are aligned and clustered based on similarity. Each fragmentation is visualized as a node (circles), and the relatedness of each node is defined by an edge (lines). Using molecular networking, structurally divergent molecules that fragment in unique ways will form their own specific cluster or constellation, whereas related molecules cluster together. Spectral information from interesting nodes is subjected to queries against the genome to identify biosynthetic gene subnetworks. By this process, the structures of molecules can be predicted or identified.

S. roseosporus is used as a strain for the industrial production of daptomycin, a cyclic lipopeptide that has been approved for the treatment of infections caused by Gram-positive bacteria, and for the treatment of bacteremia and right-sided endocarditis. The authors visualized the molecular network of S. roseosporus by MS/MS networking, and observed at least four constellations that are composed of subnetworks of MS/MS spectra with peptidic signatures. They observed nodes composed of known daptomycin variants, arylomycin variants and their biosynthetic intermediates.

Genome analysis suggested that $S$. roseosporus has the biosynthetic potential to produce napsamycins, but their production has not been reported. Molecular networking showed a constellation composed of 24 nodes, which confirmed the biosynthesis of napsamycins in this strain. In the case of another constellation with 141 nodes, peptidogenomics-guided genome mining and NMR analysis revealed the stenothricin family of molecules. The large number of nodes in the subnetwork showed a substantial number of analogs with different lipid side chains, amino-acid substitutions and glycosylated or hydrolysis products.
Furthermore, the authors demonstrated a novel mode of action of stenothricin using BioMAP antimicrobial profiles and cytological profiling. The successful identification of biosynthetic gene clusters for unreported molecules described herein highlights the importance of developing a global approach to interrogate the biosynthetic capacities of microorganisms.

The 2015 winner of the JA Medal for reviews is for an insightful review on new cycles of antibiotic discovery and development, entitled 'Prospects for new antibiotics: a molecule-centered perspective' authored by Christopher T. Walsh and Timothy A. Wencewicz. ${ }^{2}$ Unlike general encyclopedic overviews for research and development of new drugs, this thoughtful paper was particularly focused on how new chemical scaffolds and new bacterial targets could be identified and what existing targets should be re-examined for fulfilling current clinical needs.

The review begins with a description of the author's personal pathway to antibiotic research, where D-fluoroalanine was identified as a suicide substrate for alanine racemase essential for peptidoglycan assembly, and replacement of the D-Ala-D-Ala termini of the muramyl pentapeptide intermediate with D-Ala-D-Lactate was shown as the mechanism underlying vancomycin resistance in pathogenic vancomycin-resistant enterococci. The emergence of antibiotic resistance is inevitable, as the origin of the resistance genes in pathogenic bacteria derives from intrinsically resistant microbes in nature. Therefore, there is always a need for the next generation of antibiotics, but the success rate dropped rapidly after the short golden age of antibiotic discovery from nature and subsequent golden half century of medicinal chemistry. Furthermore, there has not been a new antibiotic scaffold for Gram-negative pathogens such as Klebsiella pneumoniae, Acinetobacter baumanii, Pseudomonas aeruginosa and Enterobacter species. The authors point out the importance of starting with molecular frameworks that can stand up to the rigor of development. Unfortunately, however, most antibiotic development groups and companies have chosen to reduce risk by working on known scaffolds and known targets.

The paper presents a number of promising new approaches to new scaffolds, that is, repurposing of a library built for eukaryotic kinase projects to identify hits against bacterial ATP-dependent enzymes, antisense or small interfering RNA screens that make bacterial growth sensitive to a compound that inhibits a particular pathway by lowering levels of gene expression, exploration of new niches for antibiotic-producing microorganisms such as abysses in the sea, turning on expression of silent biosynthetic gene clusters and introduction of e-DNA containing gene clusters of secondary 
metabolites into the generic host strain. More importantly, the authors discuss the promise of revisiting unexplored targets persisting in clinically validated antibacterial targets/pathways and of conducting combination therapy based on real-time diagnostics of the pathogen population in patients. This review provides profound insights into strategies for antibiotic discovery with new frameworks that are required for the fight against drug-resistant pathogens and as such is a worthy recipient of the 2015 JA Medal for reviews.

\section{CONFLICT OF INTEREST}

The authors declare no conflict of interest.

Minoru Yoshida Review Editor, The Journal of Antibiotics, Japan Antibiotics Research Association,

Tokyo, Japan E-mail: yoshidam@riken.jp

\section{ABOUT THE WINNERS}

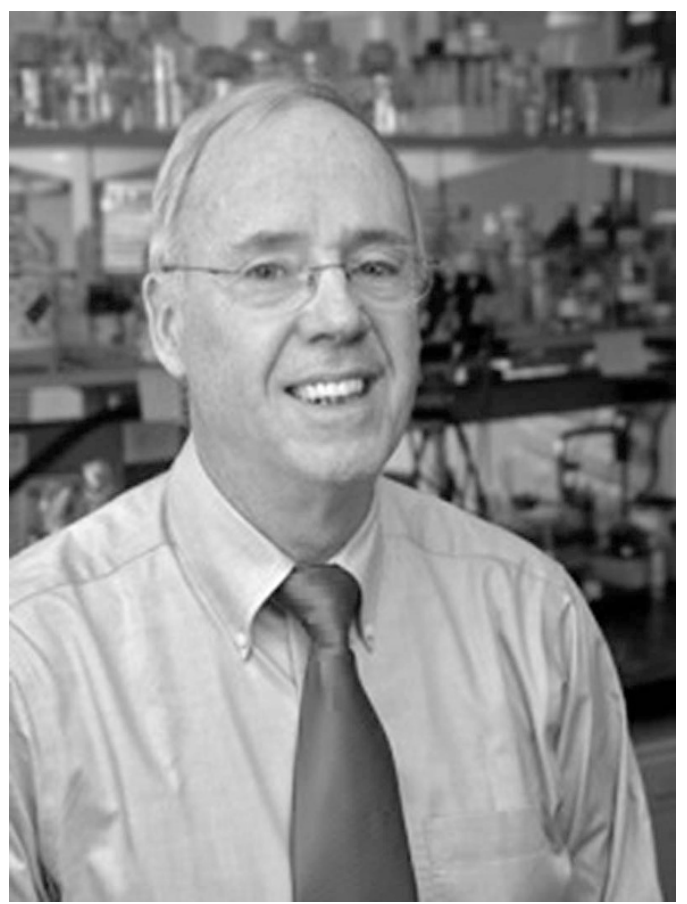

Christopher T Walsh

Christopher Walsh is Consulting Professor at the Department of Chemistry and Member, ChEM-H Institute, Stanford University, Stanford, CA, USA. His educational background is as follows: Grades 7-12: The Roxbury Latin School, Boston, MA; B.A.: Harvard College, 1965: Biology; Ph.D.: The Rockefeller University, 1970: Life Sciences; Helen Hay Whitney postdoctoral fellow, 1970-1972: Biochemistry Department, Brandeis University (Prof. R.H. Abeles). Professional experience: 1972-1987: Assistant, Associate and Full Professor of
Chemistry and Biology, MIT; 1980-1985: Uncas and Helen Whitaker Professor, MIT; 1982-1987: Chairman, MIT Chemistry Department; 1985-1987: Karl Taylor Compton Professor, MIT; 1987-1995: Chairman, Department of Biological Chemistry and Molecular Pharmacology, Harvard Medical School; 1992-1995: President, Dana-Farber Cancer Institute; 1991-2014: Hamilton Kuhn Professor of Biological Chemistry and Molecular Pharmacology, Harvard Medical School; 2010-: Senior Associate Member, The Broad Institute of Harvard and MIT and scientific advisory member to the Stanley Center at the Broad; 2014-: Emeritus Faculty, Harvard Medical School. He has been working as Consulting Professor at the Department of Chemistry, Stanford University and advisor to ChEM-H at Stanford since 2014. Awards and society memberships: Alfred P. Sloan Foundation Fellow, 1975-1977; Camille \& Henry Dreyfus Teacher-Scholar Grant Recipient, 1976-1980; Eli Lilly Award in Biochemistry, 1979; Centenary Medal \& Lectureship, Royal Society of Chemistry 1987/88; American Academy of Arts and Sciences, elected 1988; Institute of Medicine (elected 1989); National Academy of Sciences (elected 1989); Remsen Award (ACS Maryland Section) 1993; Arthur C. Cope Scholar Award, Am. Chem. Soc., 1998; Repligen Award (for Chemistry of Life Processes) Am. Chem. Soc, 1999; Bader Award (for Bioorganic Chemistry), Am Chem Soc, 2003; American Philosophical Society, elected 2003; Promega Award, American Society of Microbiology, 2004; Fritz Lipmann Medal, Am Soc Biochem \& Mol Biol, 2005; Murray Goodman Award, Am Chem Soc, 2007; Whelan Medal (U. Chicago) 2008; Ian Scott Medal, Texas A\&M, 2009; Pauling Medal and lecture, Stanford University School of Medicine, 2010; Co-recipient, 2010 Welch Award in Chemistry; Max Tishler Prize Lecture, Harvard CCB Department 2012; ICAAC Key Note Lecture 2012; Inhoffen Medal for Natural Products Chemistry 2013; Gordon Hammes Lecture, ACS, Biol Chem Division 2013; Franklin Institute Medal (Chemistry), 2014.

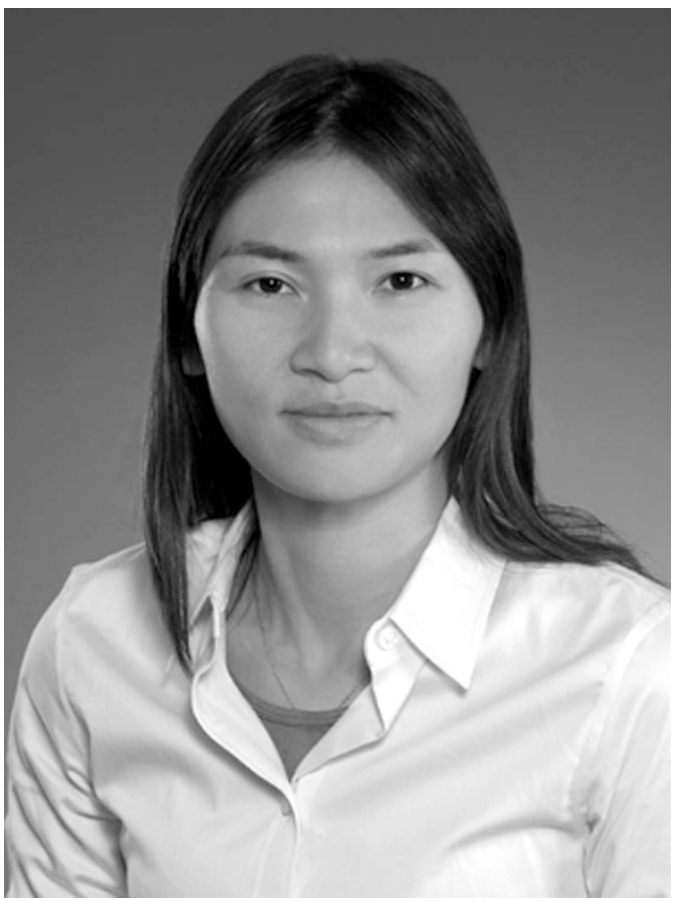

\section{Wei-Ting Liu}

Wei-Ting Liu received her PhD in 2012 in Chemistry and Biochemistry from the University of California, San Diego under the supervision of Prof. Pieter Dorrestein. During her PhD study, she worked on integrating mass spectrometric and bioinformatic approaches to investigate and characterize complex 
secondary metabolites that are involved in microbe-microbe and microbe-host interactions and demonstrated its potential to steer new platforms for therapeutic discovery. After graduation, she joined Prof. David Relman's group at Stanford University as a Life Science Research Foundation fellow to look for metabolic factors that are involved in human microbiome to gain molecular insights into how microbiota affect human diseases or health states. Currently, she works at Bayer Biologics, where she leads a metabolomics team to characterize new biological products for crop protection and crop efficiency. Dr. Liu also works in a project team together with representatives from the
Pharmaceutical and Crop Science divisions of Bayer on the implementation of a comprehensive metabolomics knowledge platform.

1 Liu, W.-T. et al. MS/MS-based networking and peptidegenomics guided genome mining revealed the stenothricin gene cluster in Streptomyces roseosporus. J. Antibiot. 67, 99-104 (2014).

2 Walsh, C. T. \& Wencewicz, T. A. Prospects for new antibiotics: a molecule-centered perspective. J. Antibiot. 67, 7-22 (2014). 